RESEARCH BRIEF

\title{
Resident Physicians' Knowledge of Breastfeeding and Infant Growth
}

\author{
Jeanne-Marie Guise, $M D, M P H$, and Gary Freed, $M D, M P H$
}

\begin{abstract}
Background: It is well documented that breastfed infants grow differently from formula-fed infants. The purpose of this study was to assess resident physicians' knowledge of breastfeeding and infant growth. Methods: A cross-sectional, self-administered survey was administered to family medicine and pediatric resident physicians from three large, hospital-based public and private programs in North Carolina. Results: One hundred and seven (46\%) of 235 residents completed the study, representing 55 percent of family medicine residents and 39 percent of pediatric residents. Ninety-nine percent of participants reported frequently or always plotting infant growth at well-child visits. None reported plotting breastfed babies on a chart specific to breastfeeding. Only 5 percent of participants knew that breastfed infants grew at a slower velocity than formula-fed infants after 4 months of age. This knowledge was not significantly related to specialty, year of training, or gender; it was significantly related to breastfeeding experience ( $\mathrm{p}<0.04)$. Of the residents who did not have personal experience with breastfeeding, 99 percent answered incorrectly compared with 88 percent of those who had some personal experience in breastfeeding. Conclusions: In this sample of family medicine and pediatric residents, almost all were unaware that breastfed infants grow at slower rates after 4 months of age. Since the frequency of breastfeeding is increasing in the United States, it is important that physicians be able to monitor the growth of breastfed infants accurately and provide expert counseling for breastfeeding mothers. (BIRTH 27:1, March 2000)
\end{abstract}

Extensive research has shown that breastmilk is the premium form of nutrition for infants. Numerous studies have reported that breastfed infants have decreased incidence of otitis media (1), diarrhea (2,3), respiratory infections $(4,5)$, urinary tract infections (6), sudden infant death syndrome (7), insulin-dependent diabetes (8), and allergic diseases (9), and may also have enhanced cognitive development (10). Breastfeeding has

Jeanne-Marie Guise is in the Department of Obstetrics and Gynecology, Oregon Health Sciences University, Portland, Oregon, and Gary Freed is in the Department of Pediatrics, University of Michigan, Ann Arbor, Michigan.

Address correspondence to Jeanne-Marie Guise, MD, MPH, Department of Obstetrics and Gynecology, Oregon Health Sciences University, Mail Code L-466, 181 SW Sam Jackson Park Road, Portland, OR 97201-3098.

(C) 2000 Blackwell Science, Inc. also been shown to benefit maternal health by decreasing the frequency of premenopausal breast cancer (11), ovarian cancer (12), and endometrial cancer (13), in addition to enhancing weight loss (14) and temporary contraception postpartum (15).

Despite the numerous beneficial health effects, in 1994 the percentage of women initiating breastfeeding in the immediate postpartum period $(60 \%)$ and continuing breastfeeding to 5 to 6 months $(23 \%)$, are well below the nation's goals of 75 percent in the immediate postpartum period and 50 percent at 5 to 6 months (16). This compares with 52 percent initiating breastfeeding in 1989, and 18.1 percent breastfeeding at 6 months (17). Factors that may contribute to early discontinuation of breastfeeding include a woman's return to the workforce (18-20), social support factors such as partners $(21,22)$, and maternal fears of milk insufficiency (23). Health caregivers can also have an effect on the initiation and duration of breastfeeding 


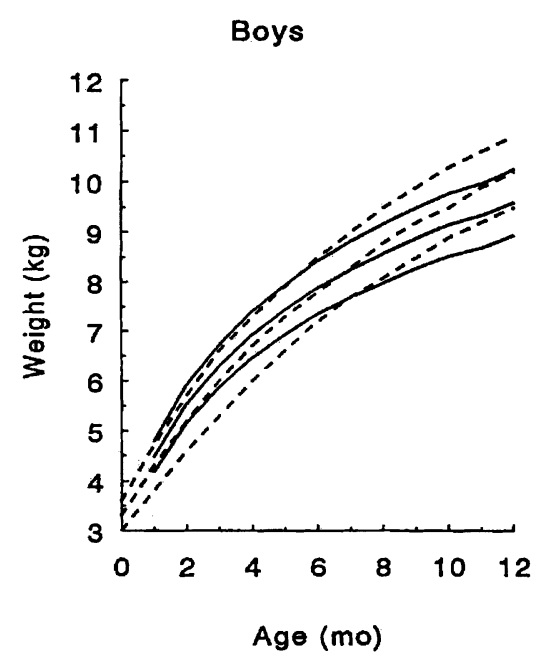

- Breast-fed set

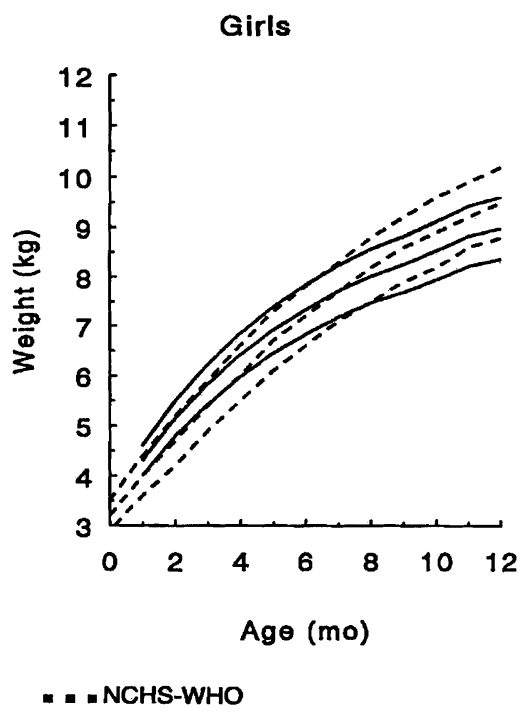

Fig. 1. Weight quartiles of "pooled" breastfed data compared with current NCHS/CDC reference. Reproduced from WHO Working Group on Infant Growth (34).

$(24,25)$. It is possible that a combination of maternal anxiety about breastmilk sufficiency, coupled with practitioner misinterpretation of normal breastfed infant growth, may contribute to early cessation of breastfeeding.

Physicians frequently use growth to evaluate the general health and nutrition of children. The most commonly used growth chart is that of the National Center for Health Statistics/Centers for Disease Control and Prevention (NCHS/CDC) (26). For children under age 2 years these growth curves are based on data from the Fels Longitudinal Study conducted in Yellow Springs, Ohio, from 1929 to 1975 (26,27). Children included in this study were largely formula-fed. Numerous studies have shown that breastfed infant growth patterns differ from the NCHS/CDC reference (28-30). Breastfed infants tend to grow at the same or increased velocity during the first 3 months of age and then more slowly after age 4 months compared with the NCHS/CDC reference (31-35). This difference in growth is most evident in weight rather than length or head circumference, making the weight-for-age and weight-for-length graphs for breastfed infants different from the NCHS/ CDC reference.

Figure 1 shows weight quartiles of breastfed infants compared with the NCHS/CDC reference in the format that is most familiar to clinicians. Figure 2 compares "pooled" breastfed mean weights versus the NCHS/ $\mathrm{CDC}$ reference. The numerical value of the $z$ score reflects the standard deviation for the "pooled" breastfed group mean when compared with the mean for the NCHS/CDC reference. Thus, if the breastfed group mean did not differ from the NCHS/CDC reference

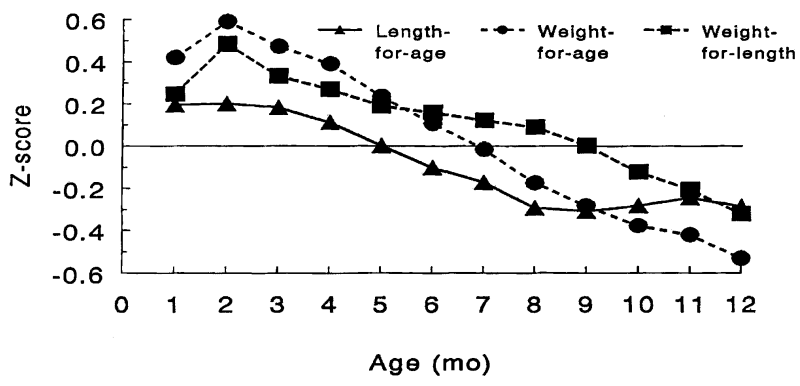

Fig. 2. Mean z score growth of "pooled" breastfed data compared with current NCHS/CDC reference. Reproduced from WHO Working Group on Infant Growth (34).

the $z$ score would be zero, whereas a $z$ score $=1$ would mean that the breastfed mean was 1 standard deviation above the NCHS/CDC reference mean. This method of comparison more clearly shows the marked difference in velocity of growth between the two groups (rather than merely a comparison of attained weight at a given time).

The purpose of this study was to assess resident physicians' knowledge of breastfeeding and infant growth.

\section{Methods}

\section{Participants}

Participants were chosen from family medicine and pediatrics-the specialties most likely to have contact with mothers of infants and toddlers. The study was 
conducted between January and March in 1997. Two hundred and thirty-five residents from three large, hospital-based public and private programs in North Carolina were eligible to participate. Of the number of eligible residents, 59 percent $(n=139)$ came from pediatrics and 41 percent $(n=96)$ from family medicine. Residents at the University of North Carolina at Chapel Hill were excluded because of potential bias from a concurrent breastfeeding study involving intensive education of residents on breastfeeding issues.

Questionnaires were self-administered and anonymous. The study was cross-sectional; questionnaires were administered at one time in each participant's training, and each facility was given one opportunity to administer the questionnaire. No incentives were given to the residents to respond. The study was approved as exempt from review by the Institutional Review Board for the University of North Carolina School of Medicine and the University of North Carolina Hospitals.

\section{Survey Design}

The questionnaire consisted of four demographic questions and 10 knowledge or clinical practice questions, using Likert scale and numerical responses when appropriate. For example, questions pertaining to growth velocity would say, "How do breastfed babies grow in weight compared with formula-fed babies at 4-7 months of age?" followed by Likert scale: 1 = slower growth, 2 = same rate of growth, 3 = faster growth, and $9=$ don't know. When numerical responses were appropriate, participants were asked, "At what month do you advise parents who are breastfeeding to introduce solid foods?" followed by a blank for a numerical answer.

The questionnaire was pretested for ambiguity and readability with convenience samples of practicing physicians and residents from various specialties. It was designed to take no more than 10 minutes to complete.

\section{Data Analysis}

Completed questionnaires were verified using the double-entry method. All data analyses were performed using STATA ${ }^{\mathrm{tm}}$ statistical software (Release 5.0, College Station, Texas). Preliminary frequency distributions were calculated for each question, and no pattern was found for missing data. Likert scale responses were collapsed into correct and incorrect responses, when appropriate, for analysis. These collapsed responses were then compared with demographic variables using chi-square analysis. Numerical responses were compared with demographic variables using one-
Table 1. Demographics of Survey Respondents $(n=107)$

\begin{tabular}{lcc}
\hline Characteristics & No. & $(\%)$ \\
\hline Specialty & & \\
$\quad$ Family medicine & 53 & $(49)$ \\
$\quad$ Pediatrics & 54 & $(51)$ \\
Year of training & & \\
$\quad$ PGY-1 & 42 & $(40)$ \\
PGY-2 & 24 & $(23)$ \\
$\quad$ PGY-3 & 38 & $(36)$ \\
Gender & & \\
$\quad$ Female & 60 & $(59)$ \\
$\quad$ Male & 42 & $(41)$ \\
Self or spouse ever breastfed a child & & \\
$\quad$ No & 83 & $(78)$ \\
$\quad$ Yes & 24 & $(22)$ \\
\hline
\end{tabular}

$P G Y=$ postgraduate year.

way analysis of variance. For all answers, $p<0.05$ was used as the measure of statistical significance.

\section{Results}

Surveys were returned by 107 of the possible 235 residents queried, representing an overall response rate of 46 percent. The response rate was higher for family medicine $(n=53,55 \%)$, than pediatric residents $(n=$ $54,39 \%)$. Respondents were fairly evenly distributed across their years of training (Table 1). More women than men responded, and only 22 percent had any personal experience with breastfeeding.

Ninety-nine percent of participants reported frequently or always plotting infant growth at well-child visits. None reported plotting breastfed babies on a growth chart specific to breastfeeding infants. Only 5 percent of participants knew that breastfed infants grew at a slower velocity than formula-fed infants after age 4 months. This knowledge was not significantly related to specialty, year of training, or gender. It was significantly related to breastfeeding experience $(p<0.04)$, with 98 percent of residents lacking personal experience with breastfeeding answering incorrectly compared with 88 percent of those who had some personal experience in breastfeeding (Table 2).

Table 2. Weight Gain $(n=107)$

\begin{tabular}{lcc}
\hline & $\begin{array}{c}\text { Weight Gain } \\
\text { Incorrect } \\
\text { Answer (\%) }\end{array}$ & $\mathrm{p}$ \\
Variables & 88 & \\
$\begin{array}{l}\text { Personal experience } \\
\quad \text { breastfeeding }\end{array}$ & 98 & \\
$\begin{array}{l}\text { No personal experience } \\
\quad \text { breastfeeding }\end{array}$ & 96 & 0.039 \\
$\begin{array}{l}\text { Male } \\
\text { Female }\end{array}$ & 95 & 0.911 \\
Family medicine & 94 & 0.712 \\
Pediatrics & 96 & \\
\hline
\end{tabular}


Pediatric residents recommended that mothers breastfeed on average for 10 months ( $\mathrm{SD} \pm 3.2$, range 1-24 mo), whereas family medicine residents recommended 11 months ( $\mathrm{SD} \pm 4.0$, range 1-24 mo); this difference was not statistically significant $(p>0.05)$. Residents who had breastfed or whose spouse had breastfed tended to recommend breastfeeding for longer(12 vs $10 \mathrm{mo}$ ), although this did not reach statistical significance $(p>0.05)$. Both groups recommended introduction of solid foods to formula-fed infants at 6 months $(S D \pm 1.7)$. Family medicine residents similarly recommended introduction of solid foods to breastfed babies at 6 months (SD \pm 2.99 ) whereas pediatric residents recommended 5 months $(\mathrm{SD} \pm 1.5)$. Neither group recommended supplementation of breastfeeding with formula. No significant relationship was found between the recommended age for introduction of solid foods and total recommended duration of breastfeeding.

\section{Discussion}

This is the first study to demonstrate a lack of knowledge by physicians-in-training about breastfeeding and infant growth. The lack was consistent across specialty, years of training, and gender. The findings are consistent with previous studies demonstrating that deficiencies in knowledge about various aspects of breastfeeding were pervasive across all specialties and year of training (36-38). The only subgroup in our study that showed a significant tendency toward awareness of growth differences of breastfed infants were physicians who had personal experience with breastfeeding.

The potential impact of this knowledge deficiency is considerable. Physicians who do not understand this difference in breastfed infant growth velocity may incorrectly label a properly growing breastfed infant as "failure to thrive" when his or her growth velocity starts to decline after age 4 months. Having made this classification error, they may then be more likely to recommend early supplementation or initiation of solid foods, either of which could discourage successful continuation of breastfeeding. This advice would increase the mother's anxieties about the adequacy of milk supply and potentially decrease confidence in her ability to breastfeed. This theoretical effect on counseling is consistent with findings by Dewey et al (35), who reported that infants starting solids at 4 to 5 months of age weighed less before 4 months of age than infants starting at 6 months or later. This suggests that weight cues may influence parental initiation of solid foods.

Since prolonged breastfeeding offers numerous maternal and infant health benefits, the American Academy of Pediatrics recommended continued breastfeeding for the first year of life (39). Yet, continuation rates for breastfeeding in the United States remain low. The 4- to 6-month window, when many physicians recommend the introduction of solid foods, is likely to be a critical time, during which mothers may be susceptible to discouraging signals from caregivers about continuation of breastfeeding. This underscores again the importance of providing accurate information and counseling to breastfeeding mothers.

This study was conducted among residents from three large hospital-based public and private programs in North Carolina. Caution must be used in generalizing the findings to smaller or community-based programs, or to programs in other parts of the country that may possess greater breastfeeding training. In addition, the response rate was only 46 percent. To standardize administration and to ensure consistency in training level at the time of survey administration, we offered each program only one opportunity to administer the questionnaire. We do not know how respondents compared with nonrespondents with respect to breastfeeding and infant growth knowledge. It is likely that respondents would be most interested in breastfeeding and therefore may present the "best case scenario" of breastfeeding knowledge.

It is clear that new growth references accounting for breastfed infants need to be developed and implemented. Until they are, it is important for residents to be educated about the differences in growth parameters between breastfed and formula-fed infants.

\section{References}

1. Saarian UM. Prolonged breastfeeding as prophylaxis for recurrent otitis media. Acad Paediatr Scand 1982;71:567-571.

2. Dewey KG, Heinig MJ, Nommsen LA. Differences in morbidity between breast-fed and formula-fed infants. $J$ Pediatr 1995; 126:696-702.

3. Howie P. Protective effect of breastfeeding against infection. BMJ 1990;300:11-16.

4. Wright AI, Holberg CJ, Martinez FD, et al. Breast-feeding and lower respiratory tract illness in the first year of life. $\mathrm{Br}$ Med J 1989;299:945-949.

5. Frank AL, Taber LH, Glezen W, et al. Breast-feeding and respiratory virus infection. Pediatrics 1982;70:239-245.

6. Cunningham AS, Jelliffe DB, Jelliffe EF. Breast-feeding and health in the 1980s: A global epidemiological review. J Pediatr 1991;118:659-666.

7. Ford RP, Taylor BJ, Mitchell EA, et al. Breastfeeding and the risk of sudden infant death syndrome. Int J Epidemiol 1993; 22:885-890.

8. Mayer EJ, Hamman RF, Gay EC, et al. Reduced risk of IDDM among breast-fed children. Diabetes 1988;37:1625-1632.

9. Lucas A, Brooke OG, Morley R, et al. Early diet of preterm infants and development of allergic or atopic disease: Randomised prospective study. BMJ 1990;300:837-840. 
10. Lucas A, Morley R, Cole TJ, et al.. Breast milk and subsequent intelligent quotient in children born preterm. Lancet 1992; 339:261-264.

11. Newcomb PA, Storer BE, Longnecker MP, et al. Lactation and a reduced risk of premenopausal breast cancer. $N$ Engl $J$ Med 1994;330:81-87.

12. Rosenblatt KA, Thomas DB, and the WHO Collaborative Study of neoplasia and steroid contraceptives. Lactation and the risk of epithelial ovarian cancer. Int J Epidemiol 1993; 22:192-197.

13. Rosenblatt KA, Thomas DB. Prolonged lactation and endometrial cancer WHO Collaborative Study of neoplasia and steroid contraceptives. Int J Epidemiol 1995;24:499-503.

14. Dewey KG, Heinig MJ, Nommsen LA. Maternal weight loss patterns during prolonged lactation. Am J Clin Nutr 1993;58: $162-166$.

15. Flynn AM. Natural family planning. Br J Fam Plann 1996; 21:146-148.

16. National Center for Health Statistics. Healthy People 2000 Review, 1995-1996. Hyattsville, MD: Public Health Service, 1996.

17. Ryan AS. The resurgence of breastfeeding in the United States. Pediatrics 1997;99: URL. http://www.pediatrics.org/cgi/content/full/99/4/e12.

18. Ryan AS, Martinez GA. Breastfeeding and the working mother: A profile. Pediatrics 1989;83:524-531.

19. Linberg LD. Women's decisions about breastfeeding and maternal employment. J Marriage Fam 1996;58:26-32.

20. Visness CM, Kennedy KI. Maternal employment and breastfeeding: Findings from the. National Maternal and Infant Health Survey. Am J Public Health 1988;997:945-950.

21. McClurg-Hitt D, Olsen J. Infant feeding decisions in the Missouri WIC program. J Hum Lact 1994;10:253-256.

22. Giugliani ERJ, Caiaffa WT, Vogelhut J, et al. Effect of breastfeeding support from different sources on mothers' decisions to breastfeed. J Hum Lact 1994;10:157-161.

23. Sjolin S, Hofvander Y, Hillervik C. Factors related to early termination of breast feeding. A retrospective study in Sweden. Acta Paediatr Scand 1977;66:505-511.

24. Kistin N, Benton D, Rao S, Sullivan M. Breastfeeding rates among black urban low-income women: Effect of prenatal education. Pediatrics 1990;86:741-746.

25. Lawrence RA. Practices and attitudes toward breast-feeding among medical professionals. Pediatrics 1982;70:912-920.

26. National Center for Health Statistics. Growth Curves for Children, Birth-18 Years, United States. Vital and Health Statis- tics, Series 11, No 165, DHEW Publication no. 78-1650. Washington, DC: Government Printing Office, 1977.

27. Hamill PVV, Drizd TA, Johnson CL, et al. Physical growth: National Center for Health Statistics percentiles. Am J Clin Nutr 1979;32:607-629.

28. Dewey KG, Heinig MJ, Nommsen LA, et al. Growth of breast-fed and formula-fed infants from 0 to 18 months: The DARLING study. Pediatrics 1992;89:1035-1041.

29. Peerson JM, Heinig MJ, Nommsen LA, et al. Use of growth models to describe patterns of length, weight, and head circumference among breast-fed and formula-fed infants: The DARLING Study. Hum Biol 1993;65:611-626.

30. Michalesen KF. Nutrition and growth during infancy: The Copenhagen Cohort Study. Acta Paediatr Suppl 1997;420: $1-31$.

31. Salmenpera L, Perheentupa J, Simes MA. Exclusively breastfed healthy infants grow slower than reference infants. Pediatr Res 1985;19:307-312.

32. Dewey KG, Heinig MJ, Nommsen LA, et al. Adequacy of energy intake among breastfed infants in the DARLING study: Relationships to growth velocity, morbidity, and activity levels. J Pediatr 1991;119:538-547.

33. Whitehead RG, Paul AA. Growth charts and the assessment of infant feeding practices in the Western world and in developing countries. Early Hum Dev 1984;9:187-207.

34. WHO Working Group on Infant Growth. An Evaluation of Infant Growth: A Summary of Analyses in Preparation for the WHO Expert Committee on Physical Status: The Use and Interpretation of Anthropometry. Publication no. WHO/NUT/ 94.8. Geneva: World Health Organization, 1994.

35. Dewey KG, Peerson JM, Brown KH, et al. Growth of breastfed infants deviates from current reference data: A pooled analysis of US, Canadian, and European data sets. Pediatrics 1995;96:495-503.

36. Freed GL, Clark SJ, Sorenson J, Lohr JA, Cefalo R, Curtis P. National assessment of physicians' breastfeeding knowledge, attitudes, training, and experience. JAMA 1995;273:472-476.

37. Mitchelman DF, Faden RR, Gielen AC, et al. Pediatricians and breast-feeding promotion: Attitudes, beliefs, and practices. Am J Health Prom 1990;4:181-186.

38. Hollen BK. Attitudes and practices of physicians concerning breast-feeding and its management. Trop Pediatr Environ Child Health 1976;22:288-293.

39. American Academy of Pediatrics Committee on Practice and Ambulatory Medicine. Pediatricians' responsibility for infant nutrition. Pediatrics 1997;99:749-751. 